\title{
User Needs and Identification of Upcoming Challenges for the Application of Planetary Information Systems
}

\author{
Stephan van Gasselt ${ }^{\mathrm{a},}$, Andrea Nass ${ }^{\mathrm{b}}$ \\ ${ }^{a}$ Geomatics Group, Department of Land Economics, National Chengchi University, Taipei, Taiwan (svg@nccu.edu.tw) \\ ${ }^{b}$ Department of Planetary Geology, German Aerospace Center (DLR), Berlin, Germany (andrea.nass@dlr.de) \\ * Corresponding author
}

Keywords: Planetary Cartography, Mapping, GIS, Planetary Sciences

\begin{abstract}
Modern geographic information systems (GIS) and related spatial information and data technology have become an integral part of planetary surface studies. In mainstream academia, GIS technology has been becoming increasingly popular since the 1990 and when compared to the developments in Earth research, GIS have experienced a somewhat delayed and slow start in the planetary sciences, despite first use dating back to the same time frame [1-3]. It can be shown that the development of GIS in the planetary sciences experiences a 10-15 years delay for a number of reasons as outlined below (see figure 1). Its introduction and final establishment was largely accelerated due to a growing community from the natural and social sciences located at Universities in Europe and the US.
\end{abstract}

While the acceptance and application of GIS in the planetary sciences have been consolidating as everyday technology in research and teaching, the range of functionalities that are commonly used is fairly limited. And functions that are actually much needed require dedicated software developments and additional resources. As a literature review shows, the range of functions in planetary research focus mainly on three aspects: (1) data management, (2) data analysis and (3) mapping and visualization, which represent the basic functions associated with modern integrated GIS concepts.

Planetary GIS gained considerable popularity for managing and arranging various datasets covering different data types, different resolutions and represented through different spatial data models using a geodetically and cartographically consistent framework to allow further analyses and visualizations. Through this function, large databases of 100s of images could be conveniently organized and arranged within a single project, either by making use of file system references or by using a more sophisticated geodatabase in the background. This capability has seen multiple development peaks over the last 30 years, including that of webGIS technology [4,6-7]. In the early 2000s data management functions were widely exploited when high-resolution Mars Orbiter Camera images at scales down to $<5$ $\mathrm{m} / \mathrm{pixel}$, the Viking MDIM mosaic at $231 \mathrm{~m} / \mathrm{px}$ and a terrain model raster representation at $463 \mathrm{~m} / \mathrm{px}$ needed to be combined. Literature suggests that GIS technology was a much needed response to this demand and helped to subsequently spawn a range of new geomorphic investigations combining terrain and image information. The other pillar of modern GIS in planetary research is contributed by classical mapping, e.g., geologic mapping and map production, quantitative morphometric studies, or more complex analyses such as age determinations through crater-size frequency analyses, or suitability analysis for landing-site studies, which again are based largely on a combination of mapping and measurement tasks [4-5]. It is difficult to identify which of these components play a larger role, as management tasks usually do not lead to publications. Whatever the exact contribution might be, the interplay of these three aspects made accessing, managing, measuring and mapping at different scales and across a range of planetary bodies extremely convenient, and today the technology can still hold up to the demands of modern datasets.

The introduction of GIS technology in planetary research has by no means been disruptive or a sudden game changer of sorts. A number of reasons might be obvious, the lack of spatial complexity in the planetary sciences being one of them. Yet another reason is certainly the critical mass of users required to help sustain the technology and to perhaps even act as a multiplier. Again other reasons are related to the composition of a user community which traditionally focussed on physics with limited contact points to the GIS developments in the Earth sciences. Research facilities on the other hand continued developing their own software for a number of reasons, including the balance between maintenance costs, extent of use, and associated outcome. For processing and analysing remote sensing data, the standard commercial off-the-shelf (COTS) as well as the free and open-source (FOS) GIS solution are still limited in terms of functions, although that line between remote sensing software and GIS becomes increasingly blurred. 


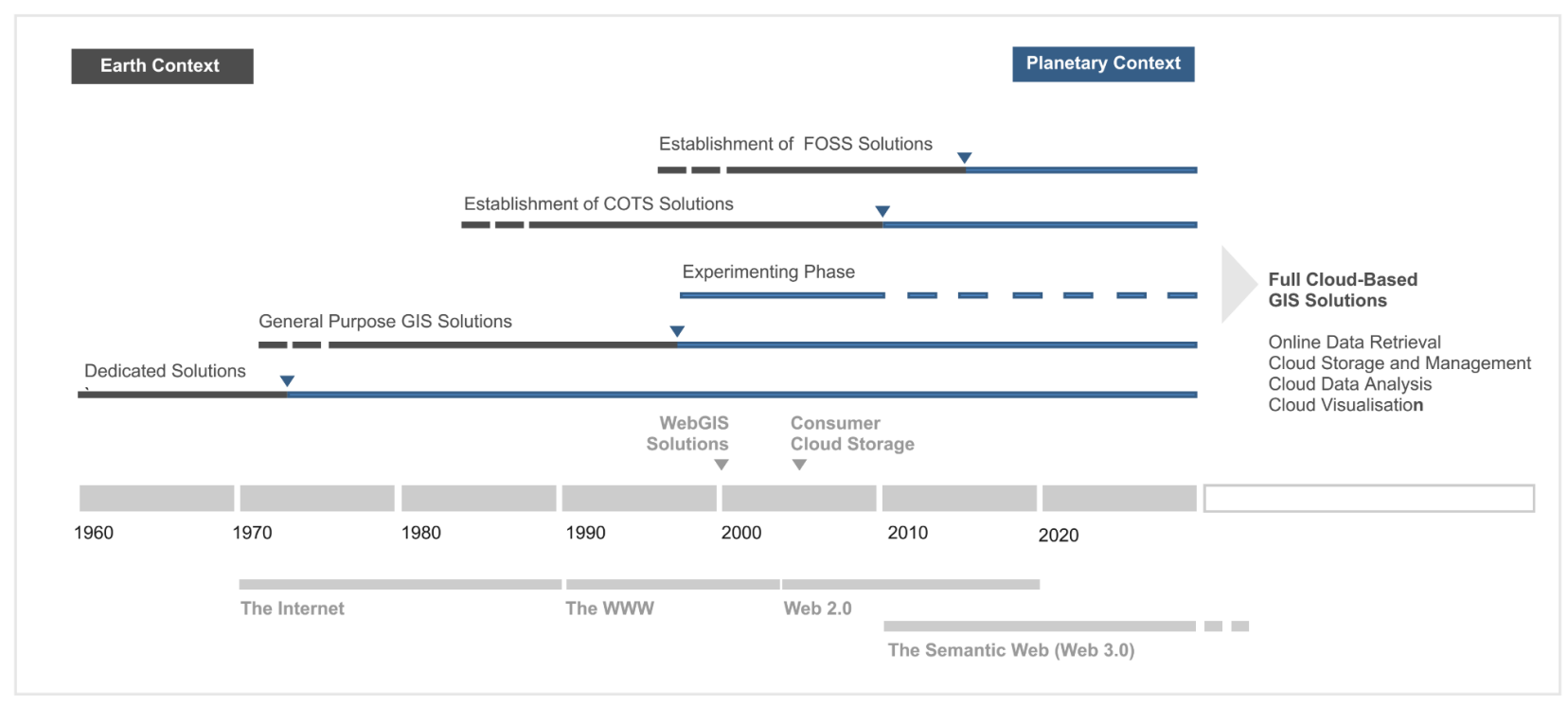

Figure 1. Time and events in application of GIS in Earth and planetary research.

This study attempts (1) to review the developments and to investigate the events and framework that caused GIS technology to develop in the planetary research domain; (2) to review the developing needs in the past, and the projected requirements in the future, based on the directions of terrestrial GIS developments; (3) to investigate the potential for GIS when it comes to complexity of analyses and how this complexity might change in the future given new long-term exploration strategies.

Some of the observations mentioned here are well backed up by 50 years of literature, some others are based on experience and observations, some have to rely on speculation as new technology developments can change associated fields immediately. While many challenges for GIS lie ahead (and most them have been put forward in early $90 \mathrm{~s}$ literature), we focus on the following aspects in this contribution: (1) the classical big data challenges of data volume and variety; (2) the need for higher sophistication of data analyses including machine learning approaches which have been slowly percolating into the research literature recently, and in particular in the light of classical engineering tasks; (3) cloud based solutions that have been targeted by industry, and the consequences for the research environment.

\section{References}

[1] Mardon, A., 1991. Utilization of geographic information system in lunar mapping. In: Abstracts of the Lunar and Planetary Science Conference XXII, Lunar and Planetary Institute (LPI), Houston, TX.

[2] Price, M. and Suppe, J., 1993. Studying Venus using a GIS database. In: Abstracts of the 24th Lunar and Planetary Science Conference, Houston, TX.

[3] Hare, T., Kirk, R., Skinner, J., Tanaka, K. 2009. Extraterrestrial GIS.- Chapter 60, 1199-1219. In: Madden, M. (ed.) Manual of Geographic Information Systems.- 1330 pp., American Society for Photogrammetry and Remote Sensing (ASPRS), Bethesda, US.

[4] Hare, T., \& Tanaka, K. 2001. Planetary Interactive GIS-on-the-Web Analyzable Database (PIGWAD), 2001. In: Proceedings of the XXth International Cartographic Conference, Lunar and Planetary Institute (LPI), Beijing.

[5] Kneissl, T., van Gasselt, S., \& Neukum, G. (2011). Map-projection-independent crater size-frequency determination in GIS environments-New software tool for ArcGIS. Planetary and Space Science, 59(11-12), 1243-1254. doi:10.1016/j.pss.2010.03.015

[6] Christensen, P.R.; Engle, E.; Anwar, S.; Dickenshied, S.; Noss, D.; Gorelick, N.; Weiss-Malik, M. 2009. JMARS - A Planetary GIS, Fall Meeting of the American Geophysical Union, \#N22A-06.

[7] Walter, S. H. G., Muller, J.-P., Sidiropoulos, P., Tao, Y., Gwinner, K., Putri, A. R. D.,, J.-R. Kim, R. Steikert, S. van Gasselt, G. G. Michael, G. Watson, B. P. Schreiner. 2018. The web-based interactive Mars analysis and research system for HRSC and the iMars project. Earth and Space Science, 5, 308-323. https://doi.org/10.1029/2018EA000389. 